\title{
The lower bound violation of shear viscosity to entropy ratio due to logarithmic correction in STU model
}

\author{
Behnam Pourhassan ${ }^{1, \mathrm{a}}$, Mir Faizal ${ }^{2,3, \mathrm{~b}}$ \\ ${ }^{1}$ School of Physics, Damghan University, Damghan, Iran \\ 2 Irving K. Barber School of Arts and Sciences, University of British Columbia-Okanagan, Kelowna, BC V1V 1V7, Canada \\ ${ }^{3}$ Department of Physics and Astronomy, University of Lethbridge, Lethbridge, AB T1K 3M4, Canada
}

Received: 22 November 2016 / Accepted: 26 January 2017 / Published online: 13 February 2017

(C) The Author(s) 2017. This article is published with open access at Springerlink.com

\begin{abstract}
In this paper, we analyze the effects of thermal fluctuations on a STU black hole. We observe that these thermal fluctuations can affect the stability of a STU black hole. We will also analyze the effects of these thermal fluctuations on the thermodynamics of a STU black hole. Furthermore, in the Jacobson formalism such a modification will produce a deformation of the geometry of the STU black hole. Hence, we use the AdS/CFT correspondence to analyze the effect of such a deformation on the dual quark-gluon plasma. So, we explicitly analyze the effect of thermal fluctuations on the shear viscosity to entropy ratio in the quark-gluon plasma, and we analyze the effects of thermal fluctuations on this ratio.
\end{abstract}

\section{Introduction}

The AdS/CFT correspondence relates the supergravity solution in the AdS space to the conformal field theory (CFT) on its boundary $[1,2]$. As the AdS/CFT correspondence relates the AdS geometry to the ground state of a conformal field theory, a deformation of the AdS solution in the bulk will also modify the CFT dual to that AdS solution. In fact, such a deformation will result in the excitation of the ground state of the dual CFT solution. So, a black hole in AdS space corresponds to heating up the system, and this in turn corresponds to exciting the ground state of the CFT. In this paper, we analyze an interesting non-extremal black hole solutions which is motivated from results obtained using the string theory, and it called the STU black hole solution $[3,4]$. The STU black holes solution can be considered as the holographic dual of quark-gluon plasma (QGP), and it is possible to study QGP using STU/CFT correspondence [5]. The

\footnotetext{
a e-mail: b.pourhassan@du.ac.ir

be-mail: f2mir@uwaterloo.ca
}

QGP is a phase in quantum chromodynamics (QCD) which exists at extremely high temperature and density. There are many important quantities in QGP such as the shear viscosity, drag force and jet-quenching and they can be calculated holographically from a STU black hole [6-8]. So, in this paper, we will first analyze a deformation of the STU black hole geometry. Then we will analyze the modification to the QGP because of such a deformation of the STU geometry using the STU/CFT correspondence. Specially, we correct the shear viscosity to entropy ratio. It is conjectures that mentioned ratio has a universal value $\frac{1}{4 \pi}$ in natural units. In fact it suggests a lower bound, so we have

$\frac{\eta}{s} \geq \frac{1}{4 \pi}$

However, due to various effects this lower bound may be violated [9-15].

In order to analyze the deformation of the STU black hole geometry by thermal fluctuations, we need to first understand the relation between geometry of a black hole and its thermodynamics. In that case, the thermodynamics of STU black holes have been studied originally by Refs. [16,17]. The area-entropy relation establishes a relation between the geometry of space-time and thermodynamics of a black hole $[18,19]$. According to the area-entropy relation the entropy of a black holes scales with the area of its horizon [20-22]. It may be noted that this observation has led to the development of the holographic principle [23,24], and AdS/CFT correspondence (which has motivated the STU/CFT or STU/QCD correspondence [5-8]) is based on the holographic principle. This is because the holographic principle related the degrees of freedom in any region of space to the degrees of freedom on the boundary surrounding that region of space. This relation between the geometry of space-time and thermodynamics is more evident in the Jacobson formalism where Einstein equation is viewed as a thermodynamics relation [25,26]. In 
fact, the Einstein equation is derived in the Jacobson formalism by requiring the Clausius relation to hold for all the local Rindler causal horizons through each space-time point. As the Jacobson formalism establishes a clear relation between the geometry of space-time and thermodynamics, quantum fluctuations in the geometry of space-time will produce thermal fluctuations in the thermodynamics of black holes in the Jacobson formalism. Thus, we expect the thermodynamics of all black holes to get corrected because of the thermal fluctuations in the Jacobson formalism.

It has been demonstrated that the area-entropy relation gets modified due to thermal fluctuations $[27,28]$. These corrections to the area-entropy relation have been studied using both analyzing the fluctuations in the energy of the system, and relating this system to a conformal field theory. However, the quantum fluctuations become important when the geometry is probed at very small scales, and the thermal fluctuations also become important when the temperature of the black hole is very large, and this corresponds to a very small size of the black hole. So, when the black hole is reduced in size due to Hawking radiation, the effects of thermal fluctuations cannot be neglected. Thus, when the size of the black hole becomes of the order of the Planck scale, the temperature of the black hole becomes very large, and the contribution from thermal fluctuations also becomes very important for such black holes. The effects of thermal fluctuations on a black hole in an anti-de Sitter space-time have been studied, and the correction to thermodynamics of such a black hole has also been obtained [29]. The corrected thermodynamics of such a black hole has been used for analyzing the phase transition in that system. The corrections to the thermodynamics of a black Saturn have also been studied, and it was observed that the entropy of both the black hole and the black ring gets corrected due to thermal fluctuations [30]. The black Saturn are thermodynamically stable because of the rotation of the black ring. However, it is possible for charged dilatonic black Saturn to remain stable because of background fields, and the thermodynamics of a charged dilatonic black Saturn has been discussed [31]. The corrections to the thermodynamics of such a charged dilatonic black Saturn have also been analyzed using the relation between this system and a conformal field theory [32]. The corrections to the thermodynamics of a modified Hayward black hole have also been discussed, and it has been demonstrated that the modified Hayward black hole is stable even after the thermal fluctuations are taken into account, as long as the event horizon is larger than a certain critical value [33]. It has been demonstrated that for all these systems the correction due to thermal fluctuations is a logarithmic correction. It may be noted that such correction terms have also been obtained from non-perturbative quantum general relativity [34], the Cardy formula [35], the exact partition function for a BTZ black hole [36], and matter fields in the backgrounds of a black hole [37-39]. In fact, even corrections obtained from string theory are logarithmic corrections [40-47]. All above studies indicated that logarithmic corrected thermodynamics of black objects is an important field of study in theoretical physics. So, in this paper, we will analyze the effect of such logarithmic corrections for a STU black hole. The logarithmic corrections to the thermodynamics of STU black hole will deform the geometry of the STU black hole in the Jacobson formalism, this will directly affect the properties of QGP inspired by the AdS/CFT correspondence. So, in this paper, we will analyze the effect of such a deformation on the shear viscosity to entropy ratio of QGP.

This paper is organized as follows. In the next section we review some important properties of STU black hole from thermodynamical point of view. In Sect. 3 we introduce logarithmic correction, and in Sect. 4 we study its effect on the shear viscosity to entropy ratio. Finally in Sect. 5 we give our conclusion.

\section{STU black hole}

In this section we recall STU model in five dimension including electric charge and write thermodynamical properties which is useful in the context of AdS/CFT correspondence. The metric for the $5 D$ STU model with three electrical charges can be written as

$\mathrm{d} s^{2}=-\frac{f_{k}}{\mathcal{H}^{\frac{2}{3}}} \mathrm{~d} t^{2}+\mathcal{H}^{\frac{1}{3}}\left(\frac{\mathrm{d} r^{2}}{f_{k}}+\frac{r^{2}}{R^{2}} \mathrm{~d} \Omega_{3, k}^{2}\right)$,

where

$$
\begin{aligned}
f_{k} & =k-\frac{\mu}{r^{2}}+\frac{r^{2}}{R^{2}} \mathcal{H}, \\
\mathcal{H} & =\prod_{i=1}^{3} H_{i}, \\
H_{i} & =1+\frac{q_{i}}{r^{2}}, \quad i=1,2,3, .
\end{aligned}
$$

Here, $R$ is the radius of the AdS space and it is related to the coupling constant as $R=1 / g$. The coupling constant is also related to the cosmological constant as $\Lambda=-6 g^{2}$. Furthermore, $r$ is the radial coordinate of the black hole, and the three electrical charges of black hole, corresponding to the three scalar field $X^{i}=\mathcal{H}^{\frac{1}{3}} / H_{i}$, are denoted by $q_{i}$, with $X^{1} X^{2} X^{3}=1($ STU $=1)$. The non-extremality parameter is denoted by $\mu$. The closed universe has $k=1$, the flat universe has $k=0$, and the open universe has $k=-1$. The metric for these universes can be written as

$$
\mathrm{d} \Omega_{3, k}^{2} \equiv\left\{\begin{array}{c}
R^{2}\left(\mathrm{~d} \rho^{2}+\sin ^{2} \rho \mathrm{d} \theta^{2}+\sin ^{2} \rho \sin ^{2} \theta \mathrm{d} \phi^{2}\right) \\
\mathrm{d} x^{2}+\mathrm{d} y^{2}+\mathrm{d} z^{2} \\
R^{2}\left(\mathrm{~d} \rho^{2}+\sinh ^{2} \rho \mathrm{d} \theta^{2}+\sinh ^{2} \rho \sin ^{2} \theta \mathrm{d} \phi^{2}\right)
\end{array}\right.
$$




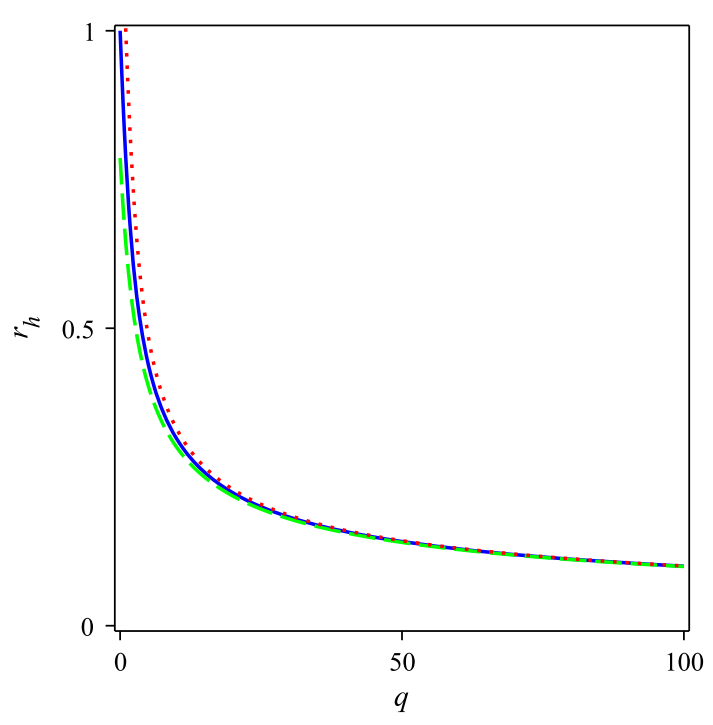

Fig. 1 Black hole event horizon in terms of the black hole charge with $R=\mu=1$ and $k=1$ (dashed line), $k=0$ (solid line), and $k=-1$ (dotted line)

for $k=1,0,-1$, respectively. We will only consider the case where there is only one-charge for the black hole $\left(q_{1}=\right.$ $\left.q, q_{2}=q_{3}=0\right)$, and where $\mathcal{H}=H=1+\frac{q}{r^{2}}$. So, the only free parameter of the model will be $q$. Now, the temperature and the entropy of this black hole can be written as [50],

$T=\frac{r_{h}}{2 \pi R^{2}} \frac{2+\frac{q+k R^{2}}{r_{h}^{2}}}{\sqrt{1+\frac{q}{r_{h}^{2}}}}$

and

$s=\frac{r_{h}^{3} \sqrt{1-\frac{q}{r_{h}^{2}}}}{4 G R^{3}}$,

where $G$ is Newton's constant and it is related to the AdS curvature as $G=\frac{\pi R^{3}}{2 N^{2}}$. Here, $N$ is the number of colors. We should note that there is a coefficient $k^{2}$ in denominator of Eq. (5) for the cases of open and closed universes. Hence, Eq. (5) is valid in its present form for all cases of $k=0, \pm 1$.

It may be noted that $r_{h}$ is given by the root of $f_{k}=0$,

$r_{h}=\frac{1}{2} \sqrt{2 \sqrt{k^{2} R^{4}+2 k q R^{2}+q^{2}+4 \mu R^{2}}-2 k R^{2}-2 q}$.

The black hole horizon is a decreasing function of the black hole charge as illustrated by Fig. 1 for different $k$. The size of the black hole will be small for large electrical charge. However, for a large value of the black hole charge there is no way to distinguish between event horizons of a black hole in open, closed, and flat universe.
Using Eqs. (4), (5), and (6), the temperature and entropy of the black hole can be expressed in terms of the black hole charge. In Fig. 2a, we demonstrate that the temperature is a decreasing function of charge for small values of $q$. There is a critical $q_{c}$, where the black hole has a minimum temperature. Then for $q>q_{c}$ the black hole temperature increases with $q$.

In Fig. 2b, we observe that by increasing the charge of the black hole, its entropy decreases. This is also expected from Fig. 1, because the entropy of the black hole is proportional to the radius of the event horizon $r_{h}$.

The specific heat can be written as

$c=T\left(\frac{\partial T}{\partial q}\right)^{-1}\left(\frac{\partial s}{\partial q}\right)$.

In Fig. 3, we plot the specific heat and observe that for the large value of the black hole charge, there is an instability. Thus, by increasing the charge of the black hole, the black hole become smaller and warmer and it enters an unstable phase. However, we do not expect such instabilities and we can remove them. The thermal fluctuations are important when the size of the black hole is small. In the next section, we will analyze the effects of the logarithmic correction, due to the thermal fluctuation, on the specific heat. We will observe that we can remove such instabilities by using such corrections.

We can use the following expression for the shear viscosity $\eta[50]:$

$\eta=\frac{r_{h}^{3} \sqrt{1+\frac{q}{r_{h}^{2}}}}{16 \pi G R^{3}}$,

to investigate famous ratio $\frac{\eta}{S}$, where $S$ is corrected entropy which will define in the next section. Thus, the conjectured universal relation $\frac{\eta}{s}=\frac{1}{4 \pi}$ hold for the STU model. So, using Eqs. (5) and (8), we can verify the universal behavior. However, there are some examples $[48,49]$ where the mentioned ratio deviates below $\frac{1}{4 \pi}$. It should be noted that the calculation of $\frac{\eta}{s}$ in the STU background was first performed by the Refs. [50,51].

\section{Thermal fluctuations}

It is possible to analyze the effects of thermal fluctuations on the black objects thermodynamics [27]. The entropy of any black objects gets corrected by a logarithmic term due to these thermal fluctuations. Thus, if we assume $\beta_{\kappa}^{-1}=T$ as a temperature close to the equilibrium, and $\beta_{0}^{-1}=T_{0}$ as the equilibrium temperature, then the corrected entropy can be written as [45]

$S=s-\frac{\ln s^{\prime \prime}}{2}$, 

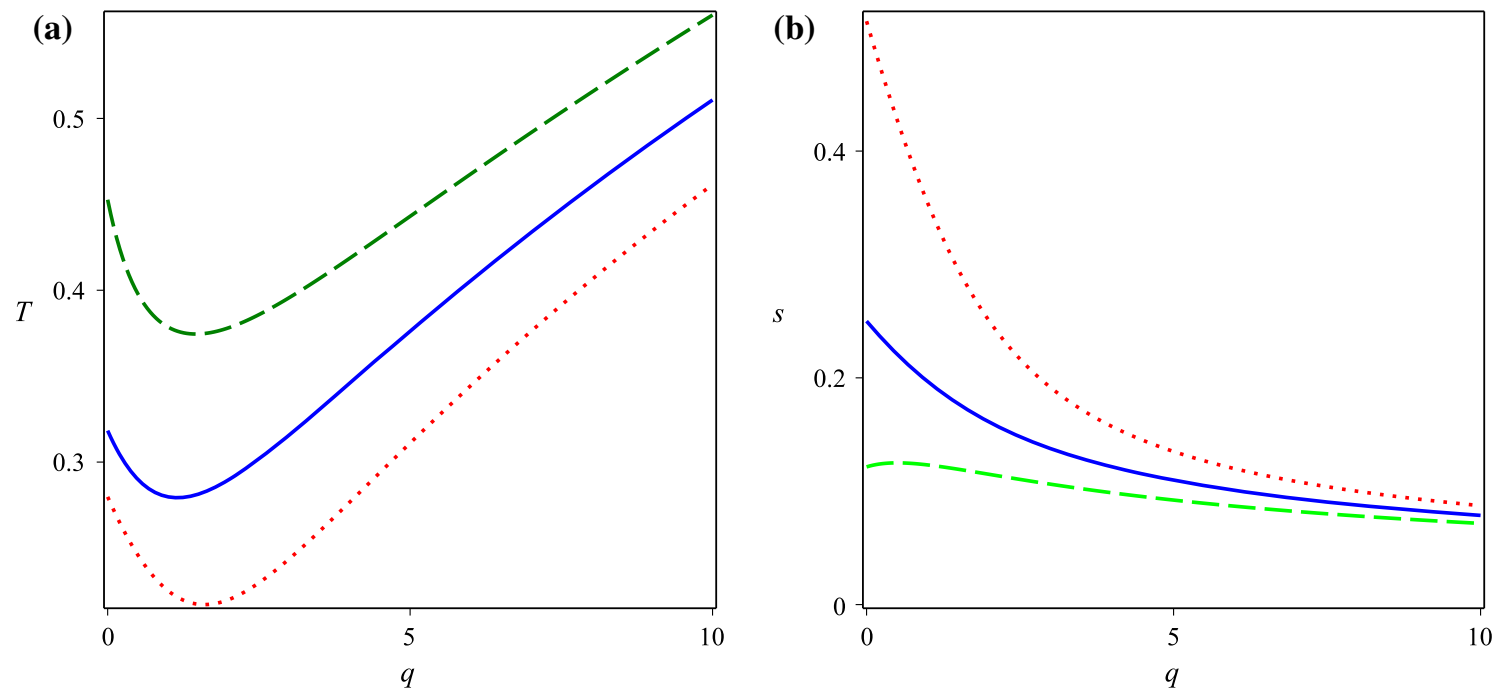

Fig. 2 Black hole temperature (a) and entropy (b) in terms of the black hole charge with $R=G=\mu=1$ and $k=1$ (dashed line), $k=0$ (solid line), and $k=-1$ (dotted line)

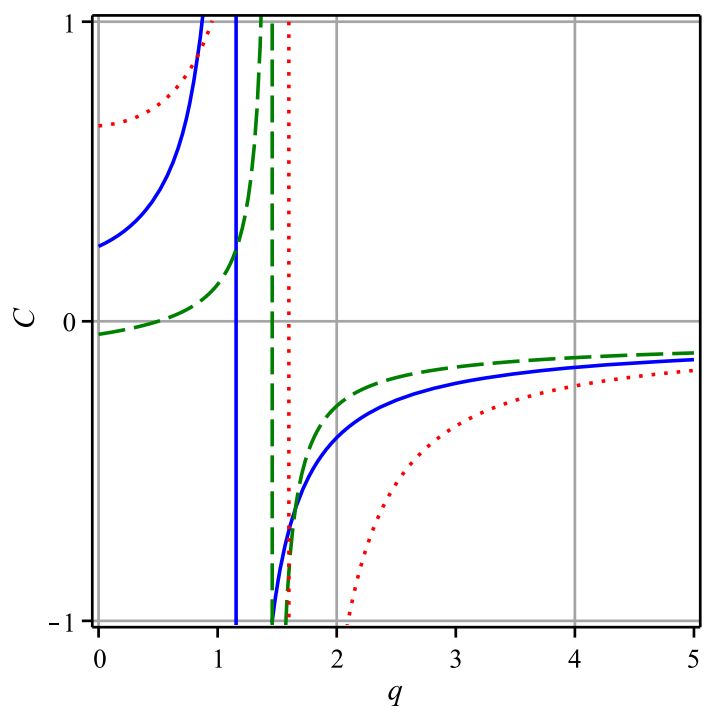

Fig. 3 Specific heat in terms of the black hole charge with $R=G=$ $\mu=1$ and $k=1$ (dashed line), $k=0$ (solid line), and $k=-1$ (dotted line)

where

$s^{\prime \prime}=\left.\left(\partial^{2} s / \partial \beta_{\kappa}^{2}\right)\right|_{\beta_{\kappa}=\beta_{0}}$.

It is also possible to express the second derivative of the entropy in terms of the fluctuations of the energy near the equilibrium. Thus, the corrected entropy can be written as $[27,30,54]$

$S=s-\frac{\alpha}{2} \ln \left|c T^{2}\right|+\cdots$,

where $s$ is the original entropy and $c$ is the original specific heat of the system. Furthermore, as almost all approaches to quantum gravity generate such a logarithmic correction, however, the coefficient of such a correction term depends on the exact model of quantum gravity that has been used. Thus, such a coefficient can be used as a parameter than can test different model of quantum gravity. This is because different approaches to quantum gravity would generate different values of the coefficient of the logarithmic term. So, in this paper, we will keep this analysis general and introduce a general parameter $\alpha$, which will be the coefficient of the logarithmic correction term. Now when $\alpha=1$, the usual thermal fluctuations taken into account, which is corresponding to a very small black object. On the other hand for $\alpha=0$, thermal fluctuations ignored, which is corresponding to the large black objects. Finally dots denote higher order corrections, which may be considered in future work.

It is also possible to relate the microscopic degrees of freedom of a black hole with a conformal field theory [32]. Thus, using the modular invariance of the partition function of the conformal field theory, corrected entropy can be written as $[27,32]$

$S=s-\frac{\alpha}{2} \ln \left|s T^{2}\right|+\cdots$.

It may be noted that, for the charged STU, there is an important difference between results obtained from Eqs. (11) and (12); however, both are the same at $q=0$. So, there is a difference between the corrections generated from a conformal field theory, and the corrections generated from the fluctuations in the energy of the system. So, we observe that the effect of thermal fluctuations for the STU black holes is different from the effect of thermal fluctuation on most other black hole solutions. This is because the correction from both these approaches generated the same effects for all the other black holes that have been analyzed using this formalism $[27,29,30,32,33,45]$. 


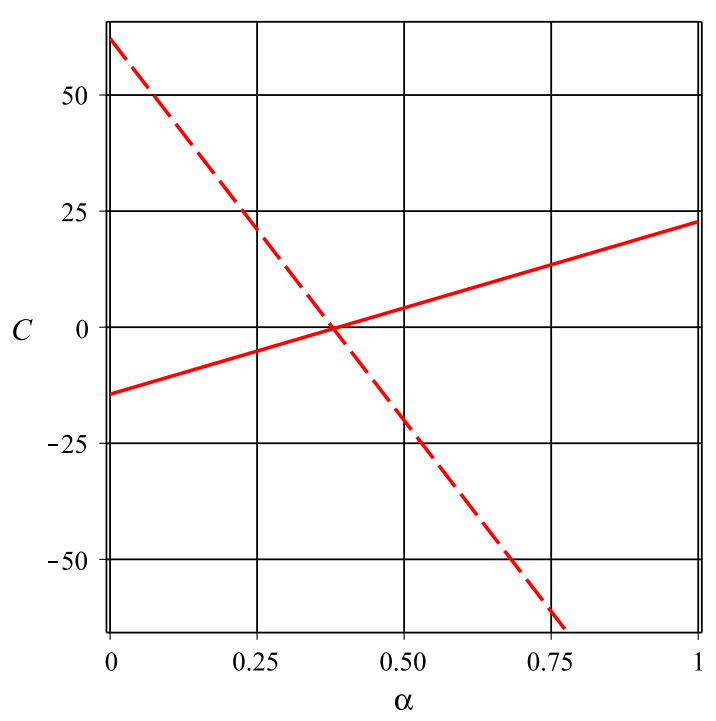

Fig. 4 Corrected specific heat in terms of $\alpha$ with $R=G=\mu=1$ and $k=0$. Dashed line denotes the case of $q<q_{c}$ and solid line denotes the case of $q \geq q_{c}$

Now using the logarithmic corrected entropy (11), we can obtain the corrected specific heat as

$C=T\left(\frac{\partial T}{\partial q}\right)^{-1}\left(\frac{\partial S}{\partial q}\right)$

In Fig. 4, we can observe the behavior of corrected specific heat for one-charged STU black hole, in terms of $\alpha$ for flat universes. For the reason we explain later, we only consider the case of $k=0$. However, we have the same situation for the open and closed universes. As we see already, some instability happened for the large black hole charge. We show that there is a critical temperature $q_{c}$ so for the case of $q>q_{c}$ the black hole is unstable, while in the presence of a logarithmic correction $(\alpha \neq 0)$ with appropriate choice of $\alpha_{c}$ the black hole is stable. For the selected values of the parameters ( $R=G=\mu=1$ ) we can see that $q_{c} \approx 1.16$ and $\alpha_{c} \approx 0.39$. A solid line of Fig. 4 shows the black hole specific heat for large electric charge. It is clear that the black hole is unstable for $\alpha=0$ as has become clear already by Fig. 3. We can see that for the appropriate choice such as $\alpha=1$ we have a totally stable black hole. Therefore we find that the logarithmic correction help to gain stability of black hole at high temperature.

\section{Shear viscosity to entropy ratio}

In this section, we are going to study the effect of the logarithmic correction on the shear viscosity to entropy ratio. We will consider three different cases corresponding to thermal fluctuation effects.

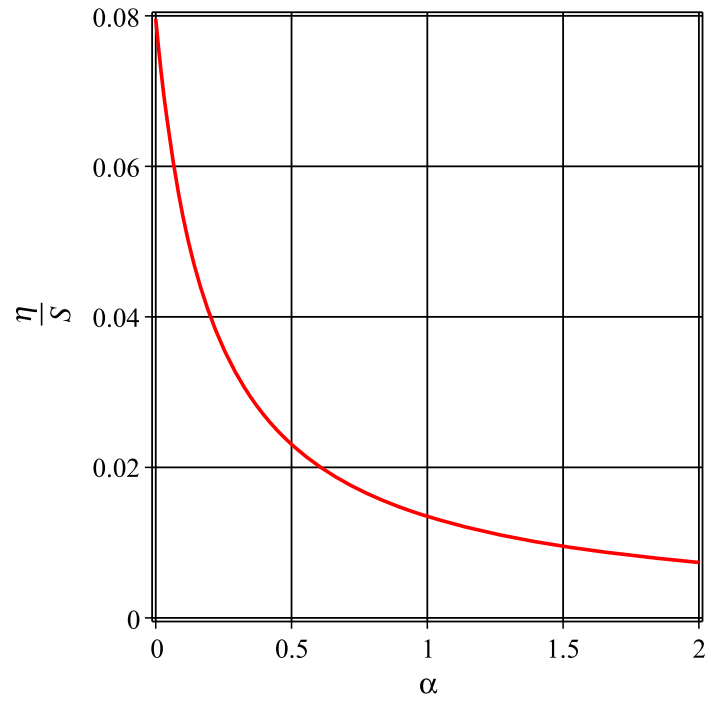

Fig. 5 Shear viscosity to entropy ratio in terms of $\alpha$ with $R=G=$ $\mu=1$ and $k=0$

So, first of all we can make a simple assumption, i.e., we can assume that the thermal fluctuations do not affect the shear viscosity, hence, we can obtain shear viscosity to entropy ratio using corrected entropy. So, we can use the corrected entropy given by Eqs. (8) and (11), to obtain the corrected shear viscosity to entropy ratio. In the case of $\alpha=$ 0 , we have $\frac{\eta}{s}=\frac{1}{4 \pi}$. However, in the presence of a logarithmic correction, we obtain

$\frac{\eta}{S}=\frac{r_{h}^{3} \sqrt{1+\frac{q}{r_{h}^{2}}}}{4 \pi r_{h}^{3} \sqrt{1+\frac{q}{r_{h}^{2}}}-8 \pi G R^{3} \alpha \ln c T^{2}}$.

So, from Fig. 5, we can observe the effect of $\alpha$ on the shear viscosity to entropy ratio. Thus, we observe that lower bound $\left(\frac{1}{4 \pi}=0.08\right)$ decreased due to the logarithmic correction. It may be noted that, using the AdS/CFT correspondence, these corrections in the bulk correspond to $1 / N^{2}$ corrections in the dual boundary theory. It is clear that $\alpha=0$ yields the conjectured universal lower bound $\left(\frac{\eta}{s}=\frac{1}{4 \pi}=0.08\right)$ while $\alpha=1$ yields $\frac{\eta}{s}=0.01-0.03$, which means universal lower bound violated. It should be noted that shear viscosity is typically defined in flat space $(k=0)$ hence in this section we only consider the case of $k=0$. As we mentioned, the logarithmic correction is correspond to $1 / N^{2}$ correction, hence $G R^{3} \alpha$ should be a small number proportional to $1 / N^{2}$ and small-alpha region of Fig. 5 is in any way reliable. Hence, we can rewrite Eq. (14) as follows:

$\frac{\eta}{S}=\frac{1}{4 \pi}+\frac{\gamma}{N^{2}} \frac{\ln c T^{2}}{r_{h}^{2} \sqrt{q+r_{h}^{2}}}$, 
where $\gamma$ is a small positive constant. It is clear that the shear viscosity to entropy ratio is a decreasing function of $\gamma$, hence the lower bound is violated.

It is possible to obtain another result by using a better and more physical approximation. In such a calculation the thermal fluctuations also correct the shear viscosity. In fact, as the corrections to the entropy are $\frac{1}{N^{2}}$ correction in the bulk, we expect such corrections to also correct the shear viscosity. It is possible to suggest the corrected value of shear viscosity, and this corrected value of the shear viscosity can correct the shear viscosity to entropy ratio. In that case, the shear viscosity to entropy ratio may be given by

$\frac{\eta}{S}=\frac{\frac{r_{h}^{3}}{4 G R^{3}} \sqrt{1+\frac{q}{r_{h}^{2}}}+\mathcal{O}(\alpha)}{4 \pi\left(\frac{r_{h}^{3}}{4 G R^{3}} \sqrt{1+\frac{q}{r_{h}^{2}}}-\frac{\alpha}{2} \ln c T^{2}\right)}$.

It is clear that the above ratio may be an increasing or a decreasing function of $\alpha$ and also may be constant for a suitable choice of $\mathcal{O}(\alpha)$, hence the lower bound for this ratio may hold. However, for the appropriate value of $\mathcal{O}(\alpha)$, lower bound may violated and the shear viscosity to entropy ratio yields zero.

The best way to calculate $\mathcal{O}(\alpha)$ is the Kubo formula, which relates the shear viscosity to the correlation function of the stress-energy tensor at zero spatial momentum by using the retarded Green function [51].

It is also possible to obtain an expression for the corrected shear viscosity, such that the ratio of the correct viscosity and corrected entropy is still does not violate conjectured universal minimum bound. This can be used to understand the behavior of shear viscosity in this limiting case. Thus, we can assume that the universal value $\frac{\eta}{S}=1 / 4 \pi$ holds for the corrected case, and we obtain

$$
\frac{\eta}{S}=\frac{\eta}{\frac{r_{h}^{3} \sqrt{1+\frac{q}{r_{h}^{2}}}}{4 G R^{3}}-\frac{\alpha}{2} \ln c T^{2}}=\frac{1}{4 \pi} .
$$

Therefore, we can obtain the corrected shear viscosity as

$$
\eta=\frac{r_{h}^{3} \sqrt{1+\frac{q}{r_{h}^{2}}}}{16 \pi G R^{3}}-\frac{1}{8 \pi} \alpha \ln c T^{2} .
$$

It may be noted that, like all gauge theories with Einstein gravity dual, a lower bound hold for all values of $\alpha$. However, by study perfect quark-gluon liquid [52], it has been found enhanced viscosity to entropy ratio $\frac{5}{8 \pi}$. It has also been found that higher curvature corrections in the dual gravitational theory modify this ratio [14], hence the higher derivative corrected STU black hole [53] is an interesting issue to investigate under logarithmic correction. It has also been argued that for certain corrected theories the lower bound is violated. Just like the logarithmic corrected case we show that the lower bound may be violated due to thermal fluctuations.

\section{Conclusion}

In this paper, we have analyzed a special case of STU black hole in five dimensions with an electric charge. We have used the AdS/CFT correspondence to investigate the effect of thermal fluctuations on the properties of QGP, specially the shear viscosity to entropy ratio. First of all we used the logarithmic corrected entropy and studied the thermodynamics of one-charged STU black hole. We found that the logarithmic corrections affect the black hole stability. For instance, instability of the black hole at high temperature changes to stable phase in the presence of a logarithmic correction. We demonstrated that the viscosity to entropy ratio of QGP dual of the STU background is reduced due to thermal fluctuations with an appropriate choice of the correction parameter. Our study was based on three different assumptions. First, we assumed that the shear viscosity not changed due to logarithmic correction and only used logarithmic correction of the entropy and found that positive value of $\alpha$ yields a violation of the lower bound. In the second assumption we write the general form of the corrected shear viscosity and claim that the appropriate value of $\alpha$ gives the lower bound violation. Finally we assumed a universal value and calculated the corrected shear viscosity. The corrections to the thermodynamics can be obtained by analyzing the fluctuations in the energy of the system. They can also be analyzed using the relation of the black hole microstates with a conformal field theory. It has been observed that the effects of thermal fluctuations from both of these approaches are the same for all black hole solutions that have been previously analyzed using this formalism $[27,29,30,32,33,45]$. However, in this paper, it was observed that the effects of thermal fluctuations for a charged STU black hole from the energy fluctuations are different from the effects of thermal fluctuations for a STU black hole obtained from conformal field theory. However, these effects are the same when the charge vanishes. It might be interesting to investigate the reason for this further.

It may be noted that a U-duality invariant expression for the area-entropy relation has been obtained for a stationary, asymptotically flat, non-extremal STU black holes [55]. It was demonstrated that this expression can be written in terms of asymptotic charges of this stationary, asymptotically flat, non-extremal STU black holes. This involves the scalar charges of the black hole which can be solved in terms of the dyonic charges and the mass of the black hole. It might also be possible to express the corrections to the area-entropy relation in such a stationary, asymptotically flat, non-extremal STU black holes using asymptotic charges. It would thus 
be interesting to analyze thermal fluctuations of such a STU black hole and discuss these corrections to the entropy using asymptotic charges. Testing quantum gravity using black objects [56] like the STU black hole is also an interesting research field.

The extended thermodynamics of a STU black hole has also been studied [57]. This was done by viewing the cosmological constant as a thermodynamic variable of the STU black hole. A fixed charge ensemble was used to perform this analysis. It was demonstrated that the phase structure associated with this black hole was conjectured to be a dual RGflow on the space of field theories. It was also observed that the phase structure of this system resembles a Van der Waals gas for certain charge configurations. Thus, for this system a family of first order phase transitions exist. Furthermore, at a critical temperature, these first order phase transitions ended in a second order phase transition. The holographic entanglement entropy for such charge configurations was also obtained. It was observed that this entanglement entropy also predicted a transition at the critical temperature. So, the entanglement entropy can be used for analyzing the system in an extended phase structure. In this analysis, holographic heat engines dual to STU black holes were also studied. It would be interesting to analyze the effect of thermal fluctuations on such a system. Thus, we could analyze the effects of thermal fluctuations on both Van der Waals gas and the holographic entanglement entropy of black holes. It would also be interesting to analyze the effects of thermal fluctuations on the holographic heat engines dual to STU black holes.

Open Access This article is distributed under the terms of the Creative Commons Attribution 4.0 International License (http://creativecomm ons.org/licenses/by/4.0/), which permits unrestricted use, distribution, and reproduction in any medium, provided you give appropriate credit to the original author(s) and the source, provide a link to the Creative Commons license, and indicate if changes were made. Funded by SCOAP ${ }^{3}$.

\section{References}

1. M. Maldacena, Adv. Theor. Math. Phys. 2, 231 (1998)

2. M. Maldacena, Int. J. Theor. Phys. 38, 1113 (1999)

3. K. Behrndt, A.H. Chamseddine, W.A. Sabra, Phys. Lett. B 442, 97 (1998)

4. K. Behrndt, M. Cvetic, W.A. Sabra, Nucl. Phys. B 553, 317 (1999)

5. B. Pourhassan, J. Sadeghi, CJP 91, 995 (2013)

6. J. Sadeghi, B. Pourhassan, JHEP 0812, 026 (2008)

7. J. Sadeghi, M.R. Setare, B. Pourhassan, S. Hashmatian, Eur. Phys. J. C 61, 527 (2009)

8. K.B. Fadafan, B. Pourhassan, J. Sadeghi, Eur. Phys. J. C 71, 1785 (2011)

9. R.C. Myers, M.F. Paulos, A. Sinha, Phys. Rev. D 79, 041901 (2009)

10. A. Adams, A. Maloney, A. Sinha, S.E. Vazquez, JHEP 0903, 097 (2009)

11. X.H. Ge, S.J. Sin, S.F. Wu, G.H. Yang, Phys. Rev. D 80, 104019 (2009)
12. X.H. Ge, S.J. Sin, JHEP 0905, 051 (2009)

13. J. Sadeghi, B. Pourhassana, A.R. Amani, Int. J. Theor. Phys. 52, $42(2013)$

14. S. Cremonini, Mod. Phys. Lett. B 25, 1867 (2011)

15. S. Cremonini, K. Hanaki, J.T. Liu, P. Szepietowski, Phys. Rev. D 80, 025002 (2009)

16. M. Cvetic, S.S. Gubser, JHEP 9904, 024 (1999)

17. A. Chamblin, R. Emparan, C.V. Johnson, R.C. Myers, Phys. Rev. D 60, 064018 (1999)

18. J.D. Bekenstein, Phys. Rev. D 7, 2333 (1973)

19. J.D. Bekenstein, Phys. Rev. D 9, 3292 (1974)

20. S.W. Hawking, Nature 248, 30 (1974)

21. S.W. Hawking, Commun. Math. Phys. 43, 199 (1975)

22. N. Altamirano, D. Kubiznak, R.B. Mann, Z. Sherkatghanad, Galaxies 2, 89 (2014)

23. L. Susskind, J. Math. Phys. 36, 6377 (1995)

24. R. Bousso, Rev. Mod. Phys. 74, 825 (2002)

25. T. Jacobson, Phys. Rev. Lett. 75, 1260 (1995)

26. R.G. Cai, S.P. Kim, JHEP 0502, 050 (2005)

27. S. Das, P. Majumdar, R.K. Bhaduri, Class. Quant. Grav. 19, 2355 (2002)

28. J. Sadeghi, B. Pourhassan, F. Rahimi, Can. J. Phys. 92, 1638 (2014)

29. B. Pourhassan, M. Faizal, Europhys. Lett. 111, 40006 (2015)

30. M. Faizal, B. Pourhassan, Phys. Lett. B 751, 487 (2015)

31. S. Grunau, Phys. Rev. D 90, 064022 (2014)

32. B. Pourhassan, M. Faizal, Phys. Lett. B 755, 444 (2016)

33. B. Pourhassan, M. Faizal, Eur. Phys. J. C 76, 145 (2016)

34. A. Ashtekar, Advanced Series in Astrophysics and Cosmology: Volume 6 Lectures on Non-Perturbative Canonical Gravity (World Scientific, 1991). http://www.worldscientific.com/worldscibooks/ $10.1142 / 1321$

35. T.R. Govindarajan, R.K. Kaul, V. Suneeta, Class. Quant. Grav. 18, $2877(2001)$

36. T.R. Govindarajan, R.K. Kaul, V. Suneeta, Class. Quant. Grav. 18, 2877 (2001)

37. R.B. Mann, S.N. Solodukhin, Nucl. Phys. B 523, 293 (1998)

38. A.J.M. Medved, G. Kunstatter, Phys. Rev. D 60, 104029 (1999)

39. A.J.M. Medved, G. Kunstatter, Phys. Rev. D 63, 104005 (2001)

40. S.N. Solodukhin, Phys. Rev. D 57, 2410 (1998)

41. A. Sen, JHEP 04, 156 (2013)

42. A. Sen, Entropy 13, 1305 (2011)

43. D.A. Lowe, S. Roy, Phys. Rev. D 82, 063508 (2010)

44. J. Jing, M.L. Yan, Phys. Rev. D 63, 24003 (2001)

45. J. Sadeghi, B. Pourhassan, M. Rostami, Phys. Rev. D 94, 064006 (2016)

46. B. Pourhassan, M. Faizal, Nucl. Phys. B 913, 834 (2016)

47. A. Pourdarvish, J. Sadeghi, H. Farahani, B. Pourhassan, Int. J. Theor. Phys 52, 3560 (2013)

48. J. de Boer, M. Kulaxizi, A. Parnachev, J. High Energy Phys. 1003, 097 (2010)

49. A. Buchel, J. Escobedo, R.C. Myers, M.F. Paulos, A. Sinha, M. Smolkin, J. High Energy Phys. 1003, 111 (2010)

50. J. Mas, JHEP 0603, 016 (2006)

51. D.T. Son, A.O. Starinets, JHEP 0603, 052 (2006)

52. H. Song, S.A. Bass, U.W. Heinz, T. Hirano, C. Shen, Phys. Rev. Lett. 106, 192301 (2011)

53. J. Sadeghi, M.R. Setare, B. Pourhassan, J. Phys. G: Nucl. Part. Phys. 36, 115005 (2009)

54. P. Pradhan, Gen. Relativ. Grav. 48, 98 (2016)

55. G. Sarosi, Phys. Rev. D 93, 024036 (2016)

56. B. Pourhassan, M. Faizal, S. Capozziello, Ann. Phys. (2017). arxiv: 1612.01359

57. E. Caceres, P.H. Nguyen, J.F. Pedraza, JHEP 1509, 184 (2015) 